\title{
Perception of Healthcare Providers in Agile Environment Imposed by the COVID-19
}

Najwa Alfarra*, Nouf Aldhawyan, Sammah Alharbi and Mohammed Sheeha

King Faisal Specialist Hospital \& RC, Physical Rehabilitation Department, Riyadh, Saudi Arabia.

*Corresponding Author: Najwa Alfarra, King Faisal Specialist Hospital \& RC, Physical Rehabilitation Department, Riyadh, Saudi Arabia. Received date: February 09, 2021; Accepted date: April 28, 2021; Published date: April 30,2021

Citation: N Alfarra, N Aldhawyan, S Alharbi and M Sheeha. (2021) Perception of Healthcare Providers in Agile Environment Imposed by the COVID-19. Clinical Research and Clinical Trials. 3(3); DOI:10.31579/2693-4779/027

Copyright: (C) 2021 Najwa Alfarra. This is an open access article distributed under the Creative Commons Attribution License, which permits unrestricted use, distribution, and reproduction in any medium, provided the original work is properly cited.

\section{Abstract:}

Background: Nearly nine months have passed since the emergence of the severe acute respiratory syndrome-coronavirus-2 (SARSCoV-2), which caused the rapidly spreading Coronavirus Disease 2019 (COVID-19) pandemic. In Saudi Arabia, unprecedented precautionary strict measures were applied to prevent virus entry to the country or to mitigate its impact when it arrives. The physical rehabilitation is the 3rd largest profession in the area of healthcare and is the most representative profession in the area of rehabilitation in Saudi Arabia. Physical rehabilitation services provide the development, maintenance, and recovery of people's movement and functional ability, improving their quality of life In this way, many hospitalized patients in the acute phase of COVID-19, as well as chronic patients, need physical rehabilitation services. This study aimed to determine the number of therapists/ clinicians who interrupted their services because of the COVID-19 pandemic and to verify the procedures adopted by the therapists/clinicians to continue serving and supporting their patients.

Methods: The sample comprised 46 therapists/clinicians who worked in King Faisal Specialist Hospital and Research Center (KFSH\&RC), 19 (41.4\%) males and 27 (58.6\%) females. The measurement instrument was an on-line survey questionnaire applied midJuly to mid-August 2020 through email. The questionnaires consisted of four primary sections. The first section gathered information on respondents' sociodemographic characteristics. The second section assessed respondents ' years of professional experience and area of specialty. The third section assessed respondents ' work load on daily bases pre/post COVID-19 crisis. The final section of the questionnaire assessed the benefits and barriers of telehealth, therapist opinion about how was the department/organization handled this pandemic issue, and to add their recommendation to improve the service.

Results: Out of the 46 therapists/clinicians, 37 (80.4\%) interrupted their work activities because of the pandemic and 9 (19.6\%) continue with the existing daily practice. The number of patients seen/day before pandemic decreased compare to pre pandemic by $21.7 \%$, in arrange of 15 patients/day to zero patient/day by $26.1 \%$, that led to change the way of therapists daily practice such as seven therapists shifted their clinics to virtual clinics, and almost most of the therapists $(n=32)$ continue working in inpatient ward, taking in their considerations the main measures that was adopted by the organization, when they deal with the direct patient contact which included: hand washing, mask use, material disinfection and gloves. The three administrative respondents work on quality issues, managerial guidelines and research. While the four orthotics/ prosthetics clinicians shifted their work to fabricate medical supportive devices to be used for the patients. Seven therapists shift their clinics to virtual visits, and they found it very convenient to the patients.

Conclusions: Our result revealed that most of the therapists/clinicians interrupted their regular work, impact number patient seen per day and face-to-face practices because of the COVID-19 pandemic, due to several reason such as spread of infection and the patients are afraid to come and they cancelled their appointment, however, half of the respondents continue with their existing work and taking into their considerations the measured that were adapted by the organization, the others who did not follow up their patients' treatment in person, most of them adapted to monitor their patients from a distance by using virtual visits.

Key words: COVID-19; physical therapists; adopted measures; pandemic; health professionals; orthotics/ prosthetics

\section{Introduction:}

In March 2020 the World Health Organization (WHO) declared the outbreak of a novel coronavirus disease (COVID-19), caused by the severe acute respiratory syndrome coronavirus 2 (SARS-CoV-2), to be a pandemic. All countries and territories of the world have reported cases of COVID-19. WHO, PAHO and public health authorities around the world are taking action to contain the spread of COVID-19 and manage its impact. Saudi Arabia was among the first countries to implement early and unprecedented precautionary measures to prevent SARS-CoV-2 introduction into the country or to mitigate its impact when it arrives; such measures were implemented early before reporting the first case in the country on March $2^{\text {nd }} 2020$. On March $8^{\text {th }}$ the Saudi government shifted schools and universities to remote learning and virtual classrooms. This was accompanied by a travel ban to all affected countries and putting in place mandatory quarantine for passengers who already arrived from 
these countries. By March $12^{\text {th }}$ all social and governmental gatherings and events were suspended or postponed including the Saudi-African and Arab-African summits. Subsequently, all international and domestic air travels, sports events, workplaces (except security and health sectors) were suspended. Digital health was quickly activated and utilized for several services such as a "my health" app that allows people to seek medical help and receive medical prescriptions without the need to visit the medical centers [1-3].

Severe acute respiratory syndrome coronavirus 2 (SARS-CoV-2) is a new coronavirus that emerged in 2019 and causes coronavirus disease 2019 (COVID-19) [2, 3]. It differs from other respiratory viruses in that it appears that human-to-human transmission occurs approximately 2 to 10 days prior to the individual becoming symptomatic [3]. The virus is transmitted from person to person through respiratory secretions. Large droplets from coughing or sneezing on surfaces within $2 \mathrm{~m}$ of the infected person. Corona virus remains viable for at least 24 hours on hard surfaces and up to 8 hours on soft surfaces [4]. The virus is transferred to another person through hand contact on a contaminated surface followed by touching the mouth, nose or eyes. Aerosol airborne infected particles created during a sneeze or cough remain viable in the air for $\leq 3$ hours. These airborne particles of COVID-19 can then be inhaled by another person or land on the mucosal membranes of the eyes.

Individuals with COVID-19 can present with an influenza-like illness and respiratory tract infection demonstrating fever (89\%), cough (68\%), fatigue (38\%), sputum production (34\%) and/or shortness of breath (19\%) [5]. The spectrum of disease severity ranged from asymptomatic infection or mild upper respiratory tract illness through to severe viral pneumonia with respiratory failure and/or death. Current reports estimate that $80 \%$ of cases were asymptomatic or mild; $15 \%$ of cases were severe (infection requiring oxygen); and 5\% were critical requiring ventilation and life support [3].

Many questions have arisen and adaptions made in the effort to respond to the crisis and the needs of our patients. The physical rehabilitation profession has been affected by the crisis, because the healthcare provider has direct contact with patients, which makes them susceptible to the transmission of infectious diseases. At the same time, consideration must be given to the fact that the physical rehabilitation profession plays a crucial role in the health of our society, and there are people in our communities whose health will be significantly impacted by disruptions to care. There were a few studies performed in that new field and they explained how COVID-19 impact daily work of the therapists/clinicians. The aim of the study to explore how therapists/clinicians in King Faisal specialist hospital at the physical rehabilitation department adapting their practice to continue serving and supporting their patients during COVID19 pandemic.
Cross-sectional study, Survey questionnaires were developed by the authors in English language. On the first page of the online questionnaire, respondents were clearly informed about the background and objectives of the study. Respondents were informed that all information and opinions provided would be anonymous and confidential. Respondents understood the completion of the survey indicates their consent of participation in the research.

The studied population consisted of therapists, and clinicians, of all genders and ages. The research inclusion criteria defined therapists/clinicians who worked in Physical Rehabilitation Department at King Faisal Specialist Hospital and Research Center (KFSH\&RC), Riyadh, Saudi Arabia. Exclusion criteria any therapists/ clinicians who did not work at King Faisal Specialist Hospital.

\section{Measurement instrument}

The measurement instrument was an on-line survey questionnaire. Data collection took place between $15^{\text {th }}$. July and $5^{\text {th }}$ August 2020 . Therapists/clinicians working in the selected hospital were e-mailed with the survey questionnaires and the survey was re-sent after 2-weeks of the initial dispatch to those participants who did not respond. All those therapists/clinicians return their completed questionnaires were included in the data analysis.

The questionnaires consisted of 20 questions in four sections. The first section gathered information on respondents' sociodemographic characteristics, included: gender, education level, work discipline, and professional title. The second section assessed respondents ' years of professional experience and area of specialty. The third section assessed respondents ' work load on daily bases pre/post COVID-19 crisis, and how is the pandemic impact the load of work per day. The final section of the questionnaire assessed the benefits and barriers of telehealth, therapist opinion about how was the department/organization handled this pandemic issue, and to add their recommendation to improve the service.

\section{Result:}

Of the 20 questionnaires delivered, the sample consisted of (59) therapists /clinicians, nevertheless, only 46 respondents completed the survey: 36 physiotherapists, 4 Orthotics/Prosthetics, and 6 Occupational therapists, with $19(41.3 \%)$ males and $27(58.7 \%)$ females. Thirty-two of the respondents had bachelor degree, twelve had master degree, and two had doctorate of healthcare management. Regarding the professional title at the KFSH\&RC three carried administrative title, 29 senior physical therapist/occupational therapists (PT/OT) therapists, nine junior PT/OT therapists, two Saudi Career Developmental Program trainers (SCDP), and three senior Orthotics/ Prosthetics. Years of practice in the profession ranged from five months to 30 years (mean $+/-16$ years). Table 1

\section{Methods:}

\begin{tabular}{|l|l|l|l|}
\hline Characteristics & $\begin{array}{l}\text { Respondents } \\
\text { educational level }\end{array}$ & $\begin{array}{l}\text { Responders } \\
\text { professional title }\end{array}$ & $\begin{array}{l}\text { Respondents professional } \\
\text { years of experience }\end{array}$ \\
\hline Bachler degree & 32 & & \\
\hline Master degree & 12 & & \\
\hline Doctorate & 2 & & \\
\hline Administrative & & 3 & \\
\hline Senior therapists & & 29 & \\
\hline Junior therapists & & 9 & \\
\hline SCDP & & 2 & 2 \\
\hline <1 Years & & & 14 \\
\hline 1-5 Years & & & 14 \\
\hline 5-10 Years & & & 16 \\
\hline$>$ 10 Years & & & \\
\hline
\end{tabular}

Table 1: Sociodemographic Characteristics and professional years of experience for the respondents. 
Third section, analyzed respondent's coverage areas: the majority of the respondents covering adult outpatient $(n=22) 47.8 \%$, followed by 18 $(39.1 \%)$ respondents' covered adult inpatient, three (6.5\%) orthotics/prosthetics outpatient respondents' two (4.3\%) pediatric inpatient, and one (2.2\%) respondent pediatric outpatient (Figure 1)
Area of coverage

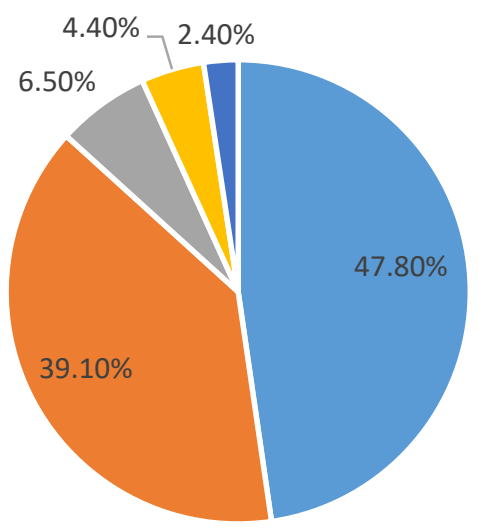

- Adult outpatient

- Pediatric inpatient
- Adult inpatient
- Pediatric outpatient

- Orth./Prosth. Outpatient

Figure 1: Respondent's area of coverage

Twenty of the respondents (43.5\%) treated between 5-10 patients per day, while 18 respondents $(39.1 \%$ ) treated between $10-15$ patients per day, six respondents $(13 \%)$ treated from 1-5 patients per day and there are two therapists $(4.4 \%)$ treated between $15-22$ patients per day in the inpatient area. Compared to post pandemic $18(39.1 \%)$ respondents treated 1-5 patients /day, ten (21.7\%) respondents treated 5-10 patients /day, eight
(17.5\%) respondents 10-15 who worked in the inpatient area, and ten $(21.7 \%)$ zero patients, because the outpatient clinics were closed and the patients were afraid to come to their visits. So $80.4 \%$ of the respondents confirmed that the pandemic affected their daily load and work while $19.6 \%$ the inpatient staff replied their work it does not affected by the pandemic.

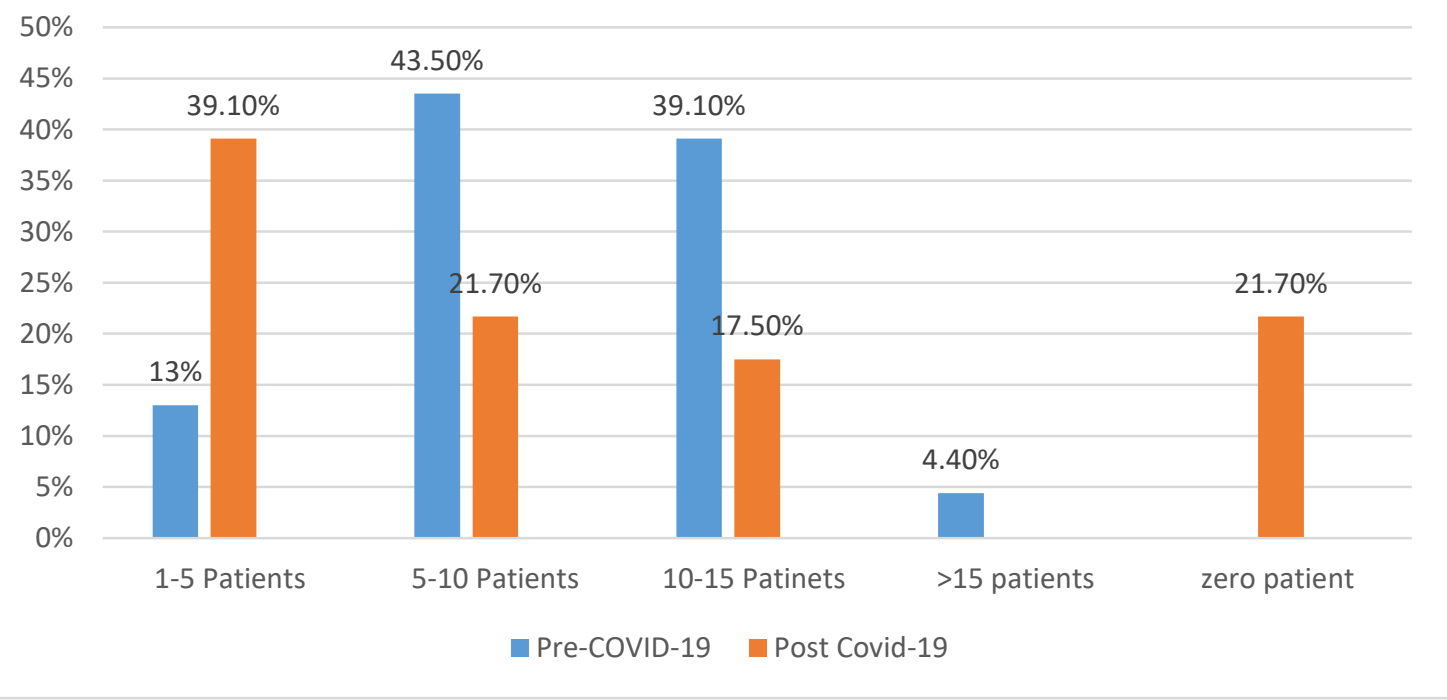

Figure 2: Number of patient seen per day during pre-versus post pandemic.

The final section of the questionnaires about the use of telehealth with the patients before the pandemic $82.6 \%$ answered they did not use before, while $17.4 \%$ they answered yes. Out of the 46 respondents, there were only seven therapists shift their clinics to virtual visits, and they found the most salient benefits that virtual visits care offers patients is convenience.
Instead of travelling to and from a hospital, or from city to another to meet the therapist, virtual visits empower people to confer with their caregivers from the comfort of their own home, makes healthcare more available, increase access of care, improve the treatment outcome, continue with the plan of care, less chance of catching any type of infection, save time, and 
cost-efficiency specially for the patients who lived outside Riyadh. While the therapists found that the virtual visits were very helpful in patient/family education only and difficulty to assess patients.

On the other hand, the therapists mentioned certain barriers of using the telehealth such as: therapists can only assess what they see and what the patient/sitter tells them, so it is difficult to assess range of motion and muscle power. Lack of technology or the knowledge to use it (especially in rural areas may present some challenges), lack of infrastructure, access to broadband or training in place to pivot to an online business model when the pandemic hit which will lead to longer treatment sessions, and possibly less efficient healthcare. However, it is not accessible for everyone, patients and doctor's skepticism, patients/careers do not prefer telehealth, difficulty with communication, and uncomfortable sharing health information via telecommunication devices specially older patients might worry more about privacy.

Additionally, the study found that $89.1 \%$ of the respondents using their free time during the pandemic by attending webinars and courses online, three respondents $6.5 \%$ reported no free time at all, and $4.4 \%$ of the respondents participated in educational activities as an organizer or speaker. Moreover, $43.5 \%$ of the respondents were strongly satisfied with the way that the physical rehabilitation department/organization handled COVID-19 lockdown and the way that the department managed the patient flow, equal percentage of the respondents were satisfied, while equal number of the respondents $6.5 \%$ dissatisfied and strongly dissatisfied, without any explanation why they were not satisfied.

When the respondents asked about the area that the organization should improve in order to maximize telehealth service to the patients during the pandemic $47.8 \%$ of the respondents replied to improve the existing telehealth, followed by $30.5 \%$ increase network speed and connectivity issues, and $21.7 \%$ work on education therapist on telehealth.

The final question it was about the therapists'/clinicians experience during COVID-19 pandemic and their concerns/recommendation to the department/organization to improve the service: A total of $20(43.5 \%)$ of the respondents had no concerns, while 8 ( $17.4 \%$ ) replied by thank you, followed by $15.2 \%$ reported that the experience was difficult for their humanity, formal psychological support should be provided by the department in a form of regular meetings to discuss their uncertainties specially at the beginning of the pandemic. Moreover, some of the respondents reported that "the departmental communication could have been better". Furthermore, $5(10.8 \%)$ respondents reported that" nothing changed about the load of the patient during COVID-19 pandemic. The only difference was the time flexibility which helped in decrease the stress of coming to the hospital on daily basis and if we come on daily basis we do not need to stay at the hospital after we finish our work and the concentration was only on completing the daily task by treating patients not the attendance". Moreover, $3(6.5 \%)$ respondents would like the virtual visits to continue after the pandemic for the long term chronic conditions that may have similar outcomes and benefits without being seen in the clinic to give the chance to others who need to be physically there. One $(2.2 \%)$ respondent reported there were huge decrease in number of patients due to utilizing more wards for COVID-19 patients, also due to stopping of elective admission, which reflect in work for few hours and that increase the quality of work. Also one $(2.2 \%)$ of the respondents confirmed that the department and the organization strictly adhered with the PPE and hand hygiene, and $2.2 \%$ of the respondents thanked the department for their endless support.

\section{Discussion}

Result of this study revealed that most therapists/ clinicians work practices were interrupted because of the COVID-19 pandemic. To minimize the impact of this public health crisis due to the new coronavirus, the physical rehabilitation department took measures, use their professional judgement to determine when, where, and how to provide care, and designed certain recommendations to assist therapist's/clinicians workforce to plan and respond to the patient's demand at the inpatient ward and outpatient clinics.

\section{Recommendation for inpatient therapists/ clinicians}

All inpatient staff should minimize surface contact, use of a respirator (like N95 respirator) instead of facemask during aerosol-generating procedures (non-invasive ventilation, intubation/extubation, nebulization, open suctioning of airway secretions), hand hygiene and personal protective equipment, respecting the proper sequence to dress (donning) (HH-gown - mask - goggles - gloves) and undress (doffing) (glovesgoggles - gown-mask- $\mathrm{HH}$ ), disposing of disposable materials when their function is complete, reusable/non-disposable materials must be carefully disinfected. All the staff should have mask fit test, staff with beard were encouraged to remove facial hair to ensure good mask fit, recurrent use of shoes cover is not recommended, as repeated removal is likely to increase the risk of staff contamination. All the therapists who covered COVID-19 ward should be aware about the role of the therapists/clinicians in the management of hospital admitted patients with confirmed COVID-19 to prevent transmission of virus. It is important for the therapists/clinicians to understand the different types of isolation rooms that exist in hospital, which is used for COVID-19 patients to prevent transmission of infection by droplet or contact routes, and should be fully aware in how to use airborne PPE precautions. The assigned therapists to cover COVID-19 ward, they were not allowed to cover the regular inpatient ward.

Recommendations for therapist's/clinicians workforce planning and preparation in outpatient clinics:

\section{Patients and staff safety:}

All patients should be treated as potential risk for COVID-19 contact transmission, therefore staffs are instructed to deal with patients accordingly. All staff should follow the hospital policy of universal masking and the use of the proper PPEs as well as maintaining the social distancing (a minimum of 1-2 meter), practice respiratory hygiene by coughing or sneezing into bent elbow or tissue and then immediately disposing of the tissue. All scheduled outpatients will be filtered and categorized according to the acuity of their condition, their overall general health and their age. Patient who fall under the high risk patient group will be given the priority in the virtual clinics unless patient physical attendance to the clinic is highly recommended and needed. Patients with acute conditions (e.g. post-operative patients, patients with acute pain and/or exacerbation of their symptoms should be served in the clinic. Outpatient staff work should be arranged on a team's basis where full staffing should not be all working on the same time. This should be achieved through the application of flexible working times, the utilization of telehealth and virtual clinics. If the employee has a fever, cough, and have difficulty breathing they should seek medical care and encourage them to stay at home until they are clear from their physicians.

\section{Safety of the work environment:}

All outpatient staff should be rotating to do actual clinics in the hospital and agile work via virtual/phone clinics from home to reduce the number of patients/staff traffic in the department. Rotating teams work arrangement will help to minimize the number of staff present in the department at any given time thus reduce the traffic in the staff designated areas (lockers, lounges charting areas). Each outpatient staff should be assigned to a specific room (clinic) which the staff will be fully responsible to clean/disinfect after each patient. Also, staff who treats their patients in the department gym will be fully responsible for cleaning/disinfecting after patient use. Floor marking and waiting areas 
segregation are applied and should be vigilantly reinforced to assure the practice of social distancing. Sitters/escorts presence in the clinic should be strictly limited to those patients who are in high need for them, no more than ONE sitter should be allowed to all patients under this category. Staff and patients should wear face mask at all times while in the clinic. The patient will not be accommodated if the patient did not comply with these instructions. Support staff: secretaries and other non-medical staff will be encouraged to work from home as much as possible. PCAs are working in flexible working hours to avoid the traffic. All the infection prevention and control guidelines disseminated to the staff by email, virtual staff meeting and signage in and around the clinic to encourage regular hand washing and use of PPE. The Majority of these measures were already disseminated by APT and WHO at the beginning of the pandemic [10].

\section{Explored the expectations of the therapists about the use of Telehealth}

\section{The definition of digital physical therapy}

Before the COVID-19 crisis, in 2017 the World Confederation for Physical Therapy (WCPT) launched a collaboration to develop initiatives to the global practice and regulations of digital physical therapy practice through a Joint WCPT/INPTRA digital physical therapy Practice Task Force (Task Force) [11]. This report published in 2019 defined digital practice as "a term used to describe health care services, support, and information provided remotely via digital communication and devices". The purpose of this initiative was "to facilitate effective delivery of physical therapy services by improving access to care and information and managing health care resources."[1] However, for several physical therapists who have never had contact with this terminology, it is important to be aware that different terms are used in this field. There is still no global well-accepted term or definition for digital physical therapy among the literature industry, policymakers, and stakeholder groups. The variety of technologies that encompass this term may include teleeducation, telemedicine, telemonitoring, teleassistance, mobile health, or Virtual visits among others, and each field has its subset of technologies and specificities [11]

At KFSH\&RC to reduce transmission and when the outpatient clinics were forced by the higher management to close, the outpatient team decided to quickly activate digital health to continue the delivery of service. There are currently no established or recognized global standards or agreement for delivering physiotherapy care digitally [1]. However, the overall emerging evidence appears to indicate that digital technologies are providing new opportunities for the physical rehabilitation profession to deliver high- quality and acceptable care to users of their service in ways that can have benefits for all ${ }^{5}$. Some national physiotherapy organizations are welcoming the use of digital practices where it enhances the service to the patient ${ }^{6}$. To implement telemedicine at the rehabilitation department (KFHS\&RC) a variety of approaches can be used such as the use of general communication tools like: email, chat/ whatsapp and virtual clinics and/or therapist's specific platforms such as online exercise prescription tools. It is important to take into account the barriers that the therapists/patients might face as a result of using the telehealth such as difficulty to access the use of these tools may present for some patients is not accessible for everyone, patients and doctor's skepticism, patients/careers do not prefer telehealth, the patient find difficulty with communication, and uncomfortable sharing health information via telecommunication devices especially older patients might worry more about privacy, so the therapist should provide support where required if possible. Additionally, the therapist might have lack of technology or the knowledge to use telehealth, lack of infrastructure, and training in place to pivot to an online business model when the pandemic hit which will lead to longer treatment sessions, and possibly less efficient healthcare, should be discussed with their manager to support them, and the most important obstacle that the therapists face; her/his inability to assess the patient. That goes with Galiano-Castillo N., et al, 2016 [19]. argued that the large-scale implementation of telehealth demands innovation in the technology market to improve capabilities and reduce cost to increase benefits. An important step for digital practice that will need support is data integration. As patients are remotely assessed or treated, professionals will need access to their relevant health data to provide the best care possible. That will help the healthcare providers to do the complete treatment after the assessment is measured [19].

That goes on line with Beatriz Minghelli et al [4]. Where they found that most physiotherapists had to interrupt their practice because of COVID19. However, since they cannot follow up with their patients' treatment in person, most physiotherapists adapted to monitor their patients from a distance using digital tools that are new to their professional activity. This was a necessary strategic measure to prevent the further spread of this pandemic. Additionally, APT, Aug.2020 added that the most important barriers are related to cost and reimbursement; legal liability, ethical issues such as confidentiality, outdated equipment, patient age and level of education; computer literacy, bandwidth range, and internet speed [10]

Furthermore, APT, Aug. 2020 [10] reported the telehealth Adoption and utilization expands prior to the pandemic, telehealth was the exception to the rule in physical therapy. Once the pandemic began changing behaviors, the number of PTs providing video consults significantly increased. However, by July only $13 \%$ of PTs providing telehealth were treating more than 10 patients per week that way, with $32 \%$ seeing fewer than one patient per week via telehealth on average. By late April, the ability for PTs to get paid for telehealth services was significantly improved, but challenges still remain: Many PTs are uncertain about payment, patient satisfaction, and outcomes. And technology challenges among patients and clinics remain a barrier to wider use. PTs should develop long-term strategies for telehealth. Telehealth has enabled PTs to treat and advise patients who otherwise might have gone without care during the public health emergency. Moving forward, PTs must ensure that they fully understand evolving regulations and best practices to ensure patient safety, privacy, and quality of care. Some patients especially those who live in rural and remote areas and those with underlying health conditions - will rely on physical therapy services delivered via telehealth. Patient access to physical therapy via telehealth must continue beyond the public health emergency [10].

Digital physical therapy offers opportunities for users, service providers, and society, such as the expansion of access to health providers or specialists, encouragement of self-management, increase of flexibility for healthcare delivery, and decrease of sick-leave duration. Treatment efficacy and patient evaluation using digital practice were already investigated for some acute and chronic musculoskeletal conditions, cardiac conditions, neurological problems, post-surgical rehabilitation, pain management, pelvic floor conditions, and respiratory dysfunctions, they found out the digital practice will not be the solution for all the challenges that physical therapist will face, however, it is an exponential growing field, widely adopted within the virus outbreak, and with the potential to reduce costs, increase quality, and overall accessibility of modern healthcare systems $[12,13,14,15,16,17,18]$.

\section{Conclusion:}

The COVID-19 pandemic presents significant challenges for the physical rehabilitation services and interrupted their regular work. This study showed that almost half of the respondents continue with their existing work especially the inpatient staff and putting in their considerations the adopted measures from WHO, and APTA, while the other therapists shifted their work either to virtual visits, or administrative, research, and quality issues. The telehealth is considered to be appropriate for any Saudi healthcare professional as well as healthcare academics and students, with much of the content having international relevance. 
Overall, the content was perceived to be useful, meaningful and appropriate to the needs of healthcare workers. We recommend that this telehealth is distributed to all healthcare workers to supplement strategic health and wellbeing provisions for employees during and after the COVID-19 pandemic. This study showed that the telehealth has high fidelity with regards to the way of care delivery, and engagement of healthcare patients, but it needs some modification such as enable therapist to do certain assessment, by this modification this system will show high usability and practicality. Further study will be needed to measure the impact of this new service on the patients, from patient's prospective view.

\section{References}

1. Abdullah A.Algaissiab, Naif Khalaf, Mazen Hassanaine, Anwar M. (2020) Preparedness and response to COVID-19 in Saudi Arabia: Building on MERS experience. Journal of Infection and Public Health. 13(6); 834-838.

2. Peter Thomas, Claire Baldwin, Bernie Bissett, Ianthe Boden, Rik Gosselink, Catherine L Granger, Carol Hodgson, Alice YM Jones, Michelle E Kho, Rachael Moses, George Ntoumenopoulos, Selina M Parry, Shane Patman, Lisa van der Lee. (2020) Physiotherapy management for COVID-19 in the acute hospital setting: clinical practice recommendations. Journal of physiotherapy. 66; 73-82.

3. Van Doremalen, Neeltje. Aerosol and Surface Stability of SARS-CoV-2 as Compared with SARS-CoV-1. The New England journal of medicine. 382(16) 1564-1567.

4. Beatriz Minghelli, Ana Soares, Andreia Guerreiro, Carolina Cabrita, Carlos Vitoria, Chloé Nunes, Claudia Martins, Diogo Gomes, Filipa Goulart, Raquel Marreiro dos Santos, Rita Antunes. (2020) Physiotherapy services in the face of a pandemic.

5. Hashem A.M., Al-Subhi T.L., Badroon N.A., Hassan A.M., Bajrai L.H.M., Banassir T.M. (2019) MERS-CoV, influenza and other respiratory viruses among symptomatic pilgrims during 2014 Hajj season. J Med Virol. 91(6):911-917.

6. Schwartz J, King CC, Yen MY. (2020) Protecting Health Care Workers during the COVID-19 Coronavirus Outbreak -Lessons from Taiwan's SARS response. Clin Infect Dis.

7. Lima CKT, Carvalho PMM, Lima IAAS, Nunes JVAO, Saraiva JS, de Souza RI, da Silva CGL, Neto MLR. (2020) The emotional impact of Coronavirus 2019-nCoV (new Coronavirus disease). Psychiatry Res.

8. Thomas P, Baldwin C, Bissett B , Boden I, Gosselink R , Granger CL , Hodgson CL , Jones AYM , Kho ME , Moses R , Ntoumenopoulos G, Parry SM , Patman S, van der Lee L (2020): Physiotherapy management for COVID-19 in the acute hospital setting. Recommendations to guide clinical practice. Version 1.0. Journal of Physiotherapy.

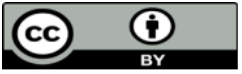

This work is licensed under Creative Commons Attribution 4.0 License

To Submit Your Article Click Here: Submit Manuscript

DOI: $10.31579 / 2693-4779 / 027$
9. Yan Y, Chen H, Chen L, Cheng B, Diao P, Dong L, Gao X, Gu H, He L, Ji C, Jin H, Lai W, Lei T, Li L, Li R, Liu D, Liu W, Lu Q, Shi Y, Song J, Tao J, Wang B, Wang G, Wu Y, Xiang L, Xie J, XuJ, Yao Z, Zhang F, Zhang J, Zhong S, Li H, Li H. (2020) Consensus of Chinese experts on protection of skin and mucous membrane barrier for health-care workers fighting against coronavirus disease. Dermatol Ther.

10. Impact of COVID-19 on the Physical Therapy Profession A Report From the American Physical Therapy Association August 2020 Update Digital Practice White Paper and Survey.

11. Scott Kruse C, Karem P, Shifflett K, Vegi L, Ravi K, Brooks M. (2018) Evaluating barriers to adopting telemedicine worldwide: a systematic review. J Telemed Telecare. 24(1):412.

12. Sjöström M., Umefjord G., Stenlund H., Carlbring P., Andersson G., Samuelsson E. (2013) Internet-based treatment of stress urinary incontinence: a randomised controlled study with focus on pelvic floor muscle training. BJU Int. 112(3):362372.

13. Bennell K.L, Nelligan R, Dobson F. (2017) Effectiveness of an internet-delivered exercise and pain-coping skills training intervention for persons with chronic knee pain: a randomized trial. Ann Intern Med.;166(7):453-462.

14. Agostini M., Moja L., Banzi R. (2015) Telerehabilitation and recovery of motor function: a systematic review and metaanalysis. J Telemed Telecare.;21(4):202-213.

15. Khan F, Amatya B, Kesselring J, Galea M. (2015) Telerehabilitation for persons with multiple sclerosis. Cochrane Database Syst Rev.

16. Cottrell M.A., Galea O.A., O’Leary S.P., Hill A.J., Russell T.G. (2017) Real-time telerehabilitation for the treatment of musculoskeletal conditions is effective and comparable to standard practice: a systematic review and meta-analysis. Clin Rehabil. 31(5):625-638.

17. Kitsiou S, Paré G, Jaana M. (2015) Effects of home telemonitoring interventions on patients with chronic heart failure: an overview of systematic reviews. J Med Internet Res. 17(3):63.

18. Hwang R, Bruning J, Morris N.R, Mandrusiak A, Russell T. (2017) Home-based telerehabilitation is not inferior to a centrebased program in patients with chronic heart failure: a randomised trial. J Physiother. 63(2):101-107.

19. Galliano-Castillo N, Cantarero-Villanueva I, Fernández-Lao C. (2016) Telehealth system: a randomized controlled trial evaluating the impact of an internet-based exercise intervention on quality of life, pain, muscle strength, and fatigue in breast cancer survivors. Cancer. 122(20):3166-3174.

Ready to submit your research? Choose Auctores and benefit from:

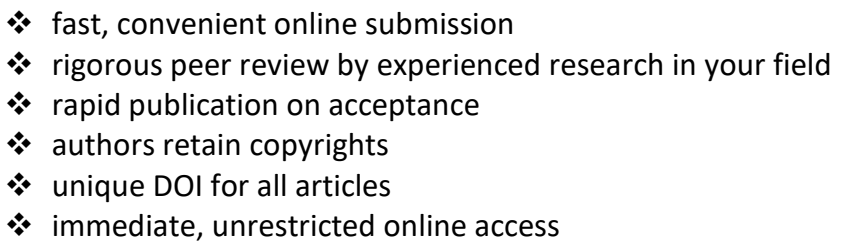

At Auctores, research is always in progress.

Learn more www.auctoresonline.org/journals/dentistry-and-oralmaxillofacial-surgery 